\title{
XyMJava System for World Wide Web Communication of Organic Chemical Structures
}

\author{
Nobuya Tanaka and Shinsaku Fujita* \\ Department of Chemistry and Materials Technology, Kyoto Institute of Technology, Matsugasaki, \\ Sakyoku, Kyoto 606-8585 Japan
}

(Received January 7, 2002; Accepted February 15, 2002)

\begin{abstract}
The XyMJava system for drawing chemical structural formulas has been developed by using the Java language in order to enhance World Wide Web communication of chemical information, where the XyM notation system proposed previously has been adopted as a language for inputting structural data. The object - oriented technique, especially the design-pattern approach, is applied to parse a XyM notation in the XyMJava system. A chemical model is introduced to encapsulate information on chemical structural formulas and used to draw the formula on a CRT display. Thereby, an HTML document containing a XyM notation can be browsed by the XyMApplet of the XyM Java system.
\end{abstract}

Key Words: XyM Notation, Java, Structural Formula, WWW

\section{Introduction}

From the early stage in the history of organic chemistry, information on chemical structures has been communicated and stored by means of systematic diagrams, i.e. chemical structural formulas. They have been linked with conventional or systematic nomenclature systems to realize oral communication as well as documentation based on printed matters such as journals and books. For example, the IUPAC nomenclature, ${ }^{1,2}$ the CAS nomenclature, ${ }^{3}$ and the HIRN nomenclature ${ }^{4,5}$ are representative as such traditional communication systems.

The exponential increase of information on chemical compounds during the two decades before 1980 had revealed the importance of chemical database, which in turn stimulated the progress of computer-manipulation of chemical structural formulas. This situation was typically exemplified by the fact that "Journal of Chemical Documentation" published by American Chemical Society was renamed into "Journal of

fujitas@chem.kit.ac.jp 
Chemical Information and Computer Sciences" in 1961. Because of the shortage of computer capacity at that time, more effective representation systems than the nomenclatures were pursued in order to enhance data storage and retrieval by computer. Because of such restriction, linear notations were developed as compressed representation systems, as summarized in excellent reviews $^{6--9}$ and books. ${ }^{10-13}$ Among them, the Dyson-IUPAC Notation, ${ }^{14,}{ }^{15}$ the Wiswesser Line-Formula Notation (WLN) ${ }^{16}$ the Hayward Notation, ${ }^{17}$ and the Skolnik Notation ${ }^{18}$ should be referred to as representatives of such data-handling notations. Since these systems aimed at unique and unambiguous nature, ${ }^{10}$ their rules were so complicated as to require a specialist to give a correct name. According to advances in computer technology, more flexible approaches such as SMILES (Simplified Molecular Input Line Entry System), ${ }^{19-21}$ the (extended) Wilcox-Levinson System, ${ }^{22}$, ${ }^{23}$ and SYBYL Line Notation ${ }^{24}$ were reported as chemical notation systems.

As a result of the explosive development of computer hardware during 1980s, the restriction due to computer capacity was removed so that more informative representation systems (topological systems) were embodied, where connection tables ${ }^{25-27}$ or equivalents were used as a key technology. ${ }^{28}$ They have been adopted by most database systems and application systems for manipulating chemical structures. ${ }^{29,30}$

Moreover, as computer hardware has further developed during the last decade (1990s), binary data or equivalent text data (e. g. Encapsulated PostScript data), which store chemical structures as image data, can be easily manipulated. Thereby, such data have been directly used in desktop publishing technique (DTP), as discussed in a review. ${ }^{31}$ In addition to the explosive development of computer hardware, the last decade has seen deep influences provided by the progress of the World Wide Web (WWW) system for the Internet, where browser techniques based on HTML (HyperText Markup Language) or XML (Extensible Markup Language) have played an important role. ${ }^{32}$ Beyond the preceding era of their storage and retrieval, the problem "how to communicate chemical structural formulas" has again attracted more and more attention as prerequisites to the WWW system. The XyM Markup Language (XyMML) has been reported by $\mathrm{us}^{33,}{ }^{34}$ as an embodiment of the spirit of markup languages, where chemical meanings such as skeletons (e. g. benzene, naphthalene, and other aromatic rings; or pyridine, quinoline, and other heteroaromatic rings), functional groups, etc. can be obtained easily from the XyMML tags. Chemical Markup Language $(\mathrm{CML})^{35}$ has been proposed for communication in the WWW system. The CML contains chemical structural data in a format equivalent to a connection table in which such chemical meanings can be obtained only by some complicated algorithms that have been adopted in the chemical database systems.

We developed the XyMTeX system for typesetting chemical structural formulas, ${ }^{36-39}$ where the XyMTeX commands were regarded as linear notation. ${ }^{40}$ Although the XyMTeX command system was adopted in a WWW browsing system, ${ }^{41}$ it required layout data in complicated cases; hence it was insufficient to the WWW communication. To avoid the drawbacks of the XyMTeX command system, we have recently reported the XyM Notation system (the left branch of Fig. 1). ${ }^{42}$

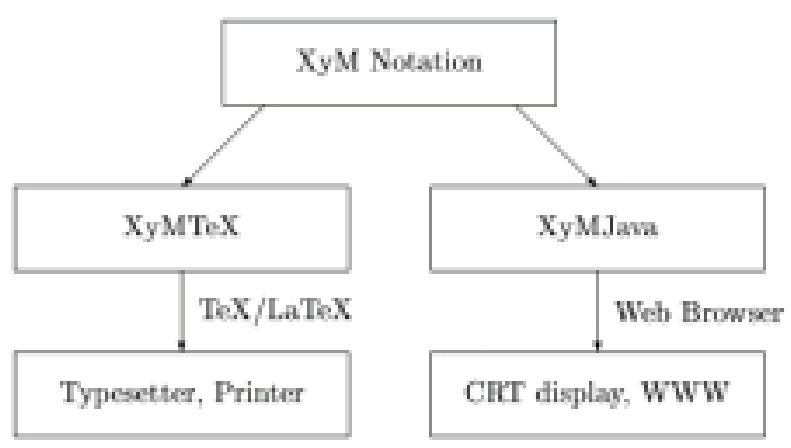

Figure 1 XyM Notation, XyMJava, and XyMTeX.

In the previous paper, ${ }^{42}$ we have shown that the XyM Notation system is one of dominant candidates capable of solving the problem "how to communicate chemical structural formulas" for the WWW communication. Since the XyM Notation system has been originally implemented as a typesetting system based on $\mathrm{TeX} / \mathrm{LaTeX}{ }^{43}$ a further device should be developed to spread the use of the XyM Notation system in the WWW. As clarified in the preceding paragraphs, our goal is the development of XyMJava ${ }^{34}$ in which the XyM Notation system is implemented by the Java language to display chemical structural formulas on a CRT screen and to communicate them via WWW (the right branch of Fig. $1)$.

\section{Minimum Features of the XуM Notation}

Since the XyMJava system of the present paper is an implementation of the XyM Notation system by the Java language, essential items on the XyM notations shall be revisited briefly. The XyM Notation system is based on the methodology that each organic compound is regarded as a derivative of a mother skeleton, where such derivation processes are categorized into (1) substitution derivation, (2) atom derivation, or (3) bond derivation. The general formats of the XyM notations are represented as follows: $¥$ IGenSkel (SKBONDLIST) [BONDLIST] \{ATOMLIST\} \{SUBSLIST\} [OMITLIST]
$¥$ FuseSkel (SKBONDLIST) [BONDLIST] \{ATOMLIST\} \{SUBSLIST\} \{FUSE\} [OMITL ¥FuseSkel (SKBONDLIST) [BONDLIST] \{ATOMLIST\} \{SUBSLIST\} \{FUSE\} [OMITI 
where the symbol $¥$ GenSkel represents a mother skeleton and the symbol ¥FuseSkel represents a fusing skeleton for constructing fused rings. They take five or six arguments with parentheses, brackets and braces.

The name of each skeleton (¥sixheterov, $¥$ sixheterovi, etc.) or each fusing skeleton (¥sixfusev, ¥sixfusevi, etc.) consists of a backslash symbol (or a yen symbol in the Japanese character system) that represents the top of a XyM notation, a stem that comes from a ring size (sixhetero etc.) or a fusion mode (sixfuse etc.) and a suffix that represents the direction of drawing the skeleton structure (v, vi, vb, vt, h, and hi).

The arguments with braces are essential ones for the skeleton $¥ G e n S k e l$ or the fusing skeleton $¥ \mathrm{FuseSkel}$. Since they cannot be omitted in any situations, an empty pair of braces \{\} is necessary in an unspecified case.

1. The argument ATOMLIST is used to bring an atom derivation, which represents the replacement of vertices by appropriate hetero atoms. The ATOMLIST contains a semicolon for delimitating every replacement modes, each of which in turn contains a double equality symbol for separating a position number from a hetero atom to be replaced. For example, the argument $\{1==0 ; 4==0\}$ in the XyM notation of 1,4-dioxane, $¥ \mathrm{sixheterov}\{1==0 ; 4==0\}\{\}$, shows that positions 1 and 4 are replaced by oxygen atoms.

2. The argument SUBSLIST is to implement a substitution derivation. For example, the argument $\{1 \mathrm{Sa}==\mathrm{F} ; 1 \mathrm{Sb}==\mathrm{F}\}$ for the XyM notation of 1,1-difluorocyclohexane, $¥ \mathrm{Sixheterov}\{\}\{1 \mathrm{Sa}==\mathrm{F} ; 1 \mathrm{Sb}==\mathrm{F}\}$, contains a semicolon to separate every substitution modes as well as a double equality symbol to distinguish between a substitution position (with a bond modifier such as ' $\mathrm{Sa}$ ' and ' $\mathrm{D}$ ' if necessary) and a substituent.

3. The argument FUSE represents a fusing bond (a lowercase or uppercase alphabet), which is omitted during a ring fusion. The usage of a lowercase or uppercase alphabet switches the normal or inverse node of the ring fusion.

The arguments with parentheses or brackets for the skeleton $¥ G e n S k e l$ or the fusing skeleton $¥ F u s e S k e l$ are optional ones that can be abbreviated if they are unnecessary:

1. Most important is the argument BONDLIST for introducing unsaturation of a skeleton as well as ring fusion (a bond derivation). The argument BONDLIST contains lowercase alphabets to designate such unsaturation, where an alphabet is assigned to each bond of the skeleton.

2. The top optional argument SKBONDLIST for treating stereochemical information. The argument SKBONDLIST contains pairs of two alphabets in braces, where each pair consists of a bond specifier (a lowercase letter) and an uppercase letter (A or B) .

3. The argument OMITLIST is a list of bond specifiers, each of which designates a bond to be deleted. As a matter of course, SKBONDLIST and OMITLIST take no common bond specifiers.

It should be noted here that any XyM notation contains arguments having specific chemical meanings such as aromatic or heteroaromatic rings (¥GenSkel and ATOMLIST), functional groups (SUBSLIST), and bond multiplicities (BONDLIST).

Any substituent can be derived from a skeleton (¥GenSkel) by adding the code ( $\mathrm{Yl}$ ) with a locant number $(n)$ to its SUBSLIST as follows:

$¥ G e n S k e 1[$ BONDLIST $]\{$ ATOMLIST $\}\{\ldots ; n==(y 1) ; \ldots\}$

where most useful arguments are selected and the other optional arguments are omitted for the sake of simplicity. This technique for generating a substituent is called "yl-function". The resulting notation for a substituent can be added to the SUBSLIST of any other skeleton. For example, cyclohexylcyclohexane is represented by the following XyM notation,

$¥$ sixheterov \{\}$\{1==¥$ sixheterov \{\}$\{4==(y 1)\}\}$

where the cyclohexyl substituent is expressed by

zixheterov \{\}$\{4==(\mathrm{y} l)\}$

A formula with a multi-nested substituent can be generated by using two or more yl-functions nested. For example, 4-(4-benzoylcyclohex-3-en-1-yl)-1-naphthol (Fig. 2) is drawn by the XyM notation:

qdecaheteroh [acfhk \{\}$\{1==\mathrm{HO} ; 4==\circ$ ¥sixheteroh [c] \{\}$\left\{1==(\mathrm{y} 1) ; 4==\frac{\circ}{0}\right.$

ftetrahedral $\{2==(\mathrm{y} 1) ; 0==\mathrm{C} ; 1 \mathrm{D}==0$;

$4==¥ \operatorname{sixheteroh}[$ ace $]\{\}\{1==($ yl $)\}\}\}$

where a percent symbol is used to designate no space in the end of a line.

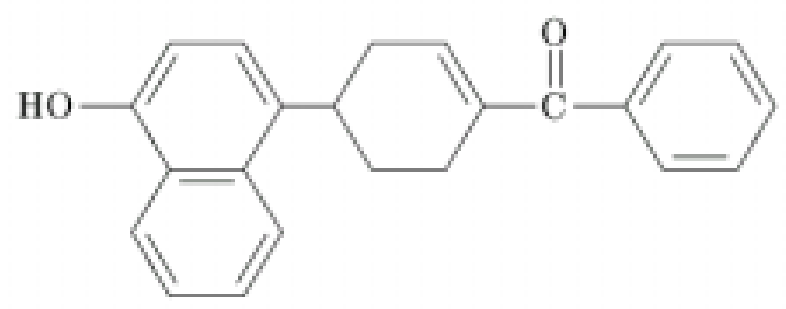

Figure 2. Formula with a multi-nested substituent.

The nesting is represented by the following scheme: (outer) ¥decaheteroh $\leftarrow$ isixheteroh $\leftarrow$ $¥$ tetrahedral $\leftarrow$ sixheteroh (inner). 
Two or more fusing skeletons ¥FuseSkel can be nested to generate a multi-fused structural formula. The structural formula of a $7 H$-pyrazino[2,3-c]carbazole can be constructed by the nesting technique in the order of 6 , $5 \leftarrow 6 \leftarrow 6$ (Fig. 3):

¥nonaheterov [begj $\{$ b¥sixfusev [ac $\{a ¥ \operatorname{sixfusev}[\mathrm{bf}]\{6==\mathrm{N} ; 3==\mathrm{N}\}\{\}\{\mathrm{D}\}\}$ ] \{\}$\{3==\mathrm{OH}\}\{\mathrm{e}\}\}]\{1==\mathrm{N}\}\{1==\mathrm{H} ; 4==\mathrm{OH}\}$

Note that a uppercase letter ' $\mathrm{D}$ ' in the FUSE argument is used in place of a lowercase letter ' $d$ ', since this usage determines the mode of fusion.<smiles>Oc1cc2[nH]c3cccc(O)c3c2c2nccnc12</smiles>

Figure 3. Multi-nested fused formula.

\section{Development of the XyMJava System}

\section{Model-View-Controller for XyMJava}

The XyMJava system for the WWW communication of information on chemical structural formulas has been developed by using the Java language. To realize an effective user interface for the XyMJava system, we have adopted the model-view-controller paradigm $(\mathrm{MVC})^{44,45}$ and design patterns for object-oriented programming. ${ }^{46,47}$

Table 1. Model-View-Controler (MVC) for XyMJava System

\begin{tabular}{|c|c|c|}
\hline MVC & Class & Function \\
\hline Model & $\begin{array}{l}\text { Chemstruct } \\
\text { Atom } \\
\text { Bond }\end{array}$ & $\begin{array}{l}\text { Representing chemical } \\
\text { structures } \\
\text { Representing atoms } \\
\text { Representing bonds } \\
\end{array}$ \\
\hline View & XyMApplet & $\begin{array}{l}\text { Displaying chemical } \\
\text { structures via a WWW } \\
\text { browser }\end{array}$ \\
\hline Controller & $\begin{array}{l}\text { XyMJava } \\
\text { ChemStructCreator } \\
\quad \text { (XyMStructCreator) } \\
\text { XyMParser } \\
\text { (XyMNotnParser) }\end{array}$ & $\begin{array}{l}\text { Main Program } \\
\text { Extraction of chemical } \\
\text { structures from XyM } \\
\text { notations } \\
\text { Parsing XyM notations } \\
\text { Auxiliary for XyMParser }\end{array}$ \\
\hline
\end{tabular}

The Java classes of the XyMJava system are listed in Table 1 in terms of the MVC paradigm. The model objects of the XyMJava system are based on a "chemical model" that is implemented as instances of the corresponding Java classes, i.e. the ChemStruct class for representing chemical structures, the Atom class for representing atoms, and the Bond class for representing bonds. The view object of the XyMJava system is based on the XyMApplet class for displaying chemical structures by using a WWW browser. The controller objects are derived from the main class XyMJava, the XyMStructCreator class for extracting chemical structures from XyM notations, the XyMParser class (and its extended class XyMNotnParser)) for parsing XyM notations, and the SubXyMParser class (and its extended class SubXyMNotnParser) for supporting auxiliaries for XyMParser.

\section{Parsing of XyM Notations for Encapsulated Structural Data}

Chemical Model as Encapsulated Structural Data

We have introduced a chemical model for the XyMJava system in order to develop a common handling technique of structural data that are applicable to other types of software than WWW software. The chemical model is illustrated as a class diagram in Fig. 4 in terms of the unified modeling language (UML), ${ }^{47}$ where a line with a rhombus represents an aggregation. For example, the Bond class multiply refers to the Atom class, while both of them are multiply referred to by the ChemStruct class.

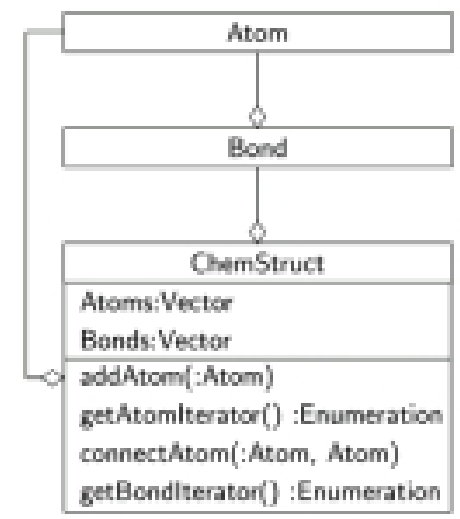

Figure 4. Class Diagram for Chemical Model.

Atom Class. The Atom class (Fig. 4) encapsulates necessary and sufficient pieces of information on an atom or a functional group, e. g. $\mathrm{x}$ - and $\mathrm{y}$-coordinates (and sometimes z-coordinate), an element symbol (String ElementType), a functional group (String AtomGroup), a flag for drawing the functional group (e.g. int RIGHT for -COOH or int LEFT for HOCO-), a flag for drawing a carbon atom $(\mathrm{C})$ on each vertex 


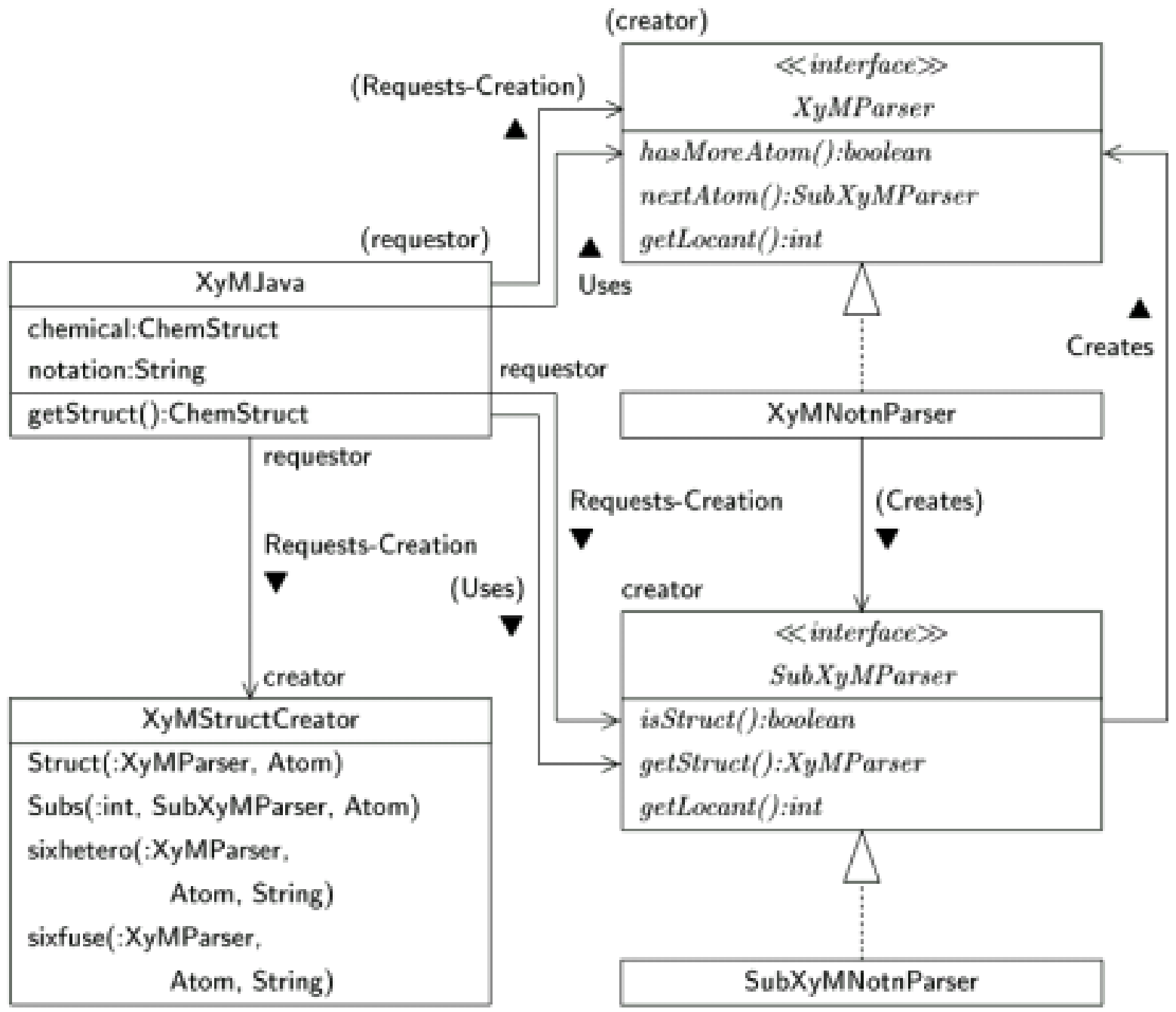

Figure 5. Class Diagram for Parsing a XyM Notation.

(boolean visible: 'false' for no drawing and 'true' for drawing C), and so on. Note that 'String' for character strings, 'int' for integers, and 'boolean' for boolean values are default classes provided by the Java packages.

Bond Class. The Bond class (Fig. 4) encapsulates necessary and sufficient pieces of information on a bond, e.g. a bond multiplicity (int bondType), terminal atoms (Atom beginAtom and Atom endAtom), incident positions (int beginAtomConnectDirection for a beginning atom and int endAtomConnectDirection for a ending atom), an adjustment flag for a double bond (int right: 'rightHand' for an endocyclic double bond), and so on.

ChemStruct. The ChemStruct class (Fig. 4) has no concrete information on atoms and bonds but contains a set of methods for referring to Atom objects (e. g. void addAtom(:Atom) for setting 'Atoms.addElement' to a Vector instance Atoms, an Enumeration instance getAtomlterator() for getting 'Atoms.elements()') as well as another set of methods for referring to Bond objects (e.g. void connectAtom(:Atom, Atom) for setting 'Bonds.addElement' to a Vector instance Bonds, and an Enumeration instance getBondlterator() for getting 'Bonds.elements()').

Parsing of XyM Notations. To parse a XyM notation, the XyMParser class and its subclass SubXyMParser have been developed as interfaces (abstract classes), as shown in a class diagram based on UML (Fig. 5).

They have been respectively implemented as concrete classes, XyMNotnParser and SubXyMNotnParser, which contain concrete codes for parsing a XyM notation. According to the UML formats, an open triangle represents with a dotted line represents a generalization (inversely implementation), and an arrow with a solid line represents a navigability, and a solid triangle on the arrow line represents a relationship (e.g. Requests-Creation) between the roles of participant classes (e. g. requestor and creator).

The XyMParser class is an interface for dividing a XyM notation into a set of arguments, i.e., a skeleton name $¥ G e n S k e l$ or $¥ G e n F u s e)$, a bond list 
(BONDLIST), an atom list (ATOMLIST), a substitution list (SUBSLIST), etc. This process is called a dividing process for the sake of simplicity. The SubXyMParser class is an interface for subdividing each of the components into sub-arguments, where the atom list, for example, is divided into a locant number, a bond modifier, an atom, etc. This process is called a subdividing process. Since the abstract classes, XyMParser and SubXyMParser, behave as interfaces, any change of the concrete classes, XyMNotnParser and SubXyMNotnParser, into other concrete classes (e.g. XyMMLParser for parsing XyM Markup Language) gives no influences on the XyMStructCreator class that creates structural formulas.

Figure 5 shows that the interfaces (XyMParser and SubXyMParser) and the concrete classes (XyMNotnParser and SubXyMNotnParser) for parsing a XyM notation can be regarded as a dual embodiment of the Factory-Method pattern. ${ }^{46,} 47$ This dual embodiment can be applied recursively to treat nesting structures in a substituent list (SUBSLIST) and in a bond list (BONDLIST). The Iterator pattern ${ }^{46,47}$ is also used to treat branches in a tree structure produced from a XyM notation.

To parse the arguments of a XyM notation (a dividing process), the XyMJava class uses the XyMParser class as a Product, where the XyMJava class (as a requestor) is linked with the SubXyMParser class (as a creator) in a Requests-Creation fashion. Thereby, the SubXyMParser class as a FactoryIF (factory interface), or more precisely the corresponding concrete Factory SubXyMNotnParser, creates the XyMParser class as a 'Product'. As a result, the XyMNotnParser class is regarded as a concrete Product. As found in the code listed in Fig. 6, the SubXyMParser class contains the getStruct() method as a XyMParser instance in order to get a nested structure.

On the other hand, the XyMJava class uses the SubXyMParser class that is switched into a Product in a subdividing process designated with parentheses in Fig. 5. Thus, the XyMJava class (as a requestor) requests the creation of the XyMParser class (as a creator), which acts as a FactoryIF (factory interface) in this process so as to make the corresponding concrete Factory XyMNotnParser create the SubXyMParser class as a 'Product'. As a result, the corresponding implement SubXyMNotnParser is now regarded as a concrete Product. As found in the code listed in Fig. 6, the XyMParser class contains the getFuseStruct() method as a XyMParser instance in order to get a fusing structure. The XyMParser class also contains the nextAtom() method for getting a next atom and the nextSubs() method for getting a next substituent, where both the methods are SubXyMParser instances.

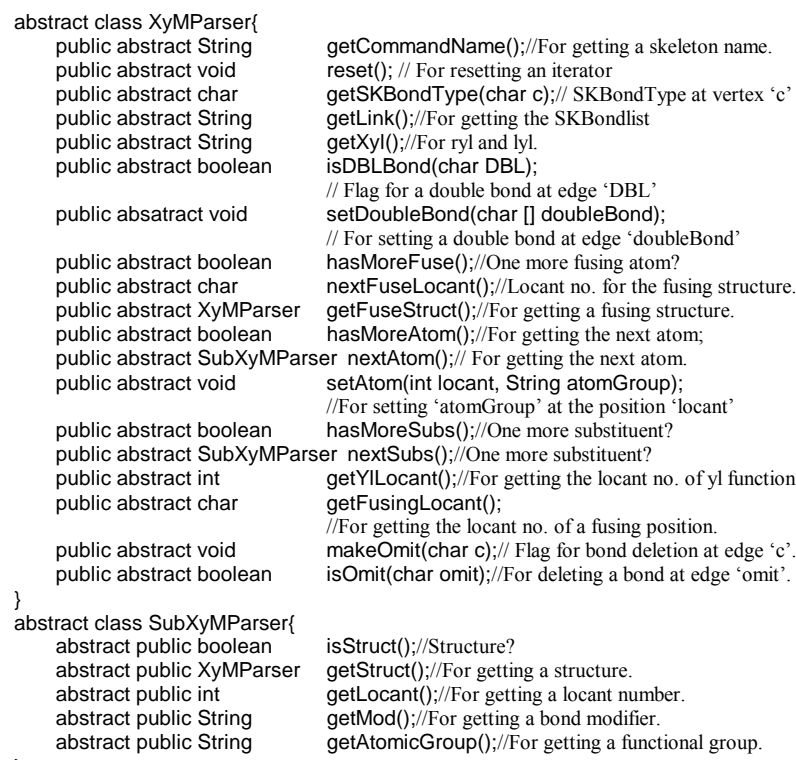

Figure 6. Codes of XyMParser and SubXyMParser Class.

\section{Creation of a Chemical Model}

The class diagram of the XyMJava package is shown in Fig. 7. 


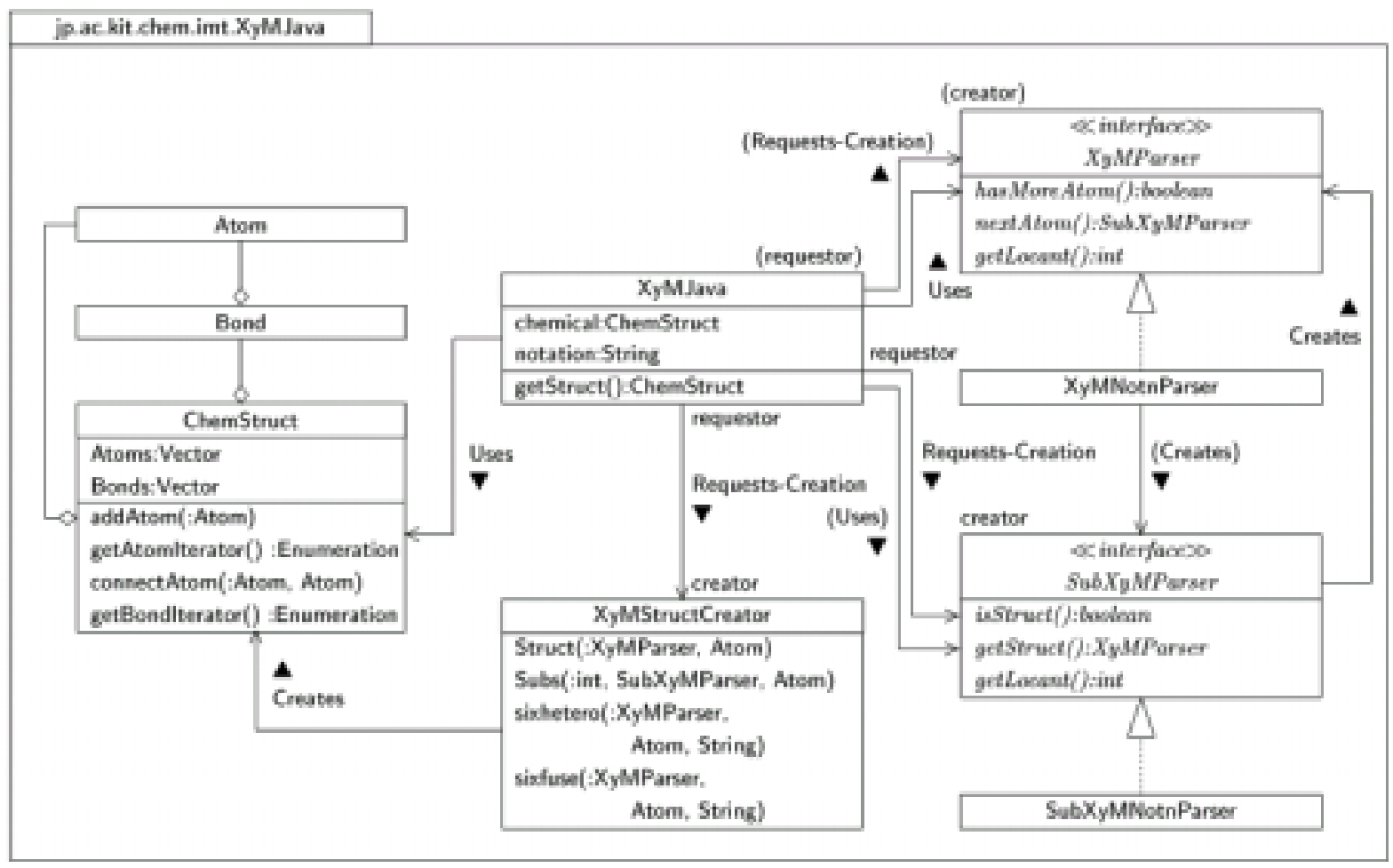

Figure 7. Class Diagram of the XyMJava Package.

The XyMStructCreator class leaves the parsing of a XyM notation to XyMParser and SubXyMParser and has access to a chemical model produced from the XyM notation. The XyMStructCreator class contains methods for creating respective skeletons (¥GenSkel); for example, the sixhetero method is to create $¥$ sixhetrov, ¥sixhetrovi, ¥sixhetroh, and $¥$ sixhetrohi. Each of these method calls the Struct method recursively to treat a nested structure of the XyM notation.

\section{Displaying Structural Formulas}

To display a chemical structural formula, we use the XyMApplet class that has been developed as an Applet instance of Java. The XyMApplet class uses the XyMJava class that has been collected with the other classes to give a package jp.ac.kit.chem.imt.XyMJava, as shown in Fig. 8.

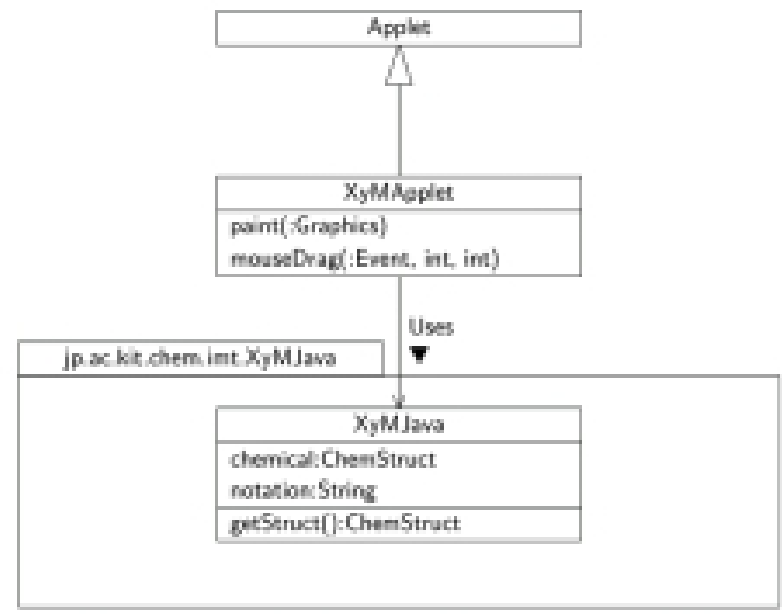

Figure 8. Class Diagram of the XyMApplet.

The XyMApplet class works as follows:

1. It gets a XyM notation from a HTML document via a WWW browser.

2. It sends the XyM notation to the XyMJava class for parsing.

3. The resulting chemical model is used to display the corresponding chemical structural formula by means of a WWW browser.

4. The formula on display can be enlarged, reduced, and moved by mouse clipping or dragging. 


\section{Use of the XyMJava System}

Figure 9 illustrates an example of a XyM notation in an HTML document.

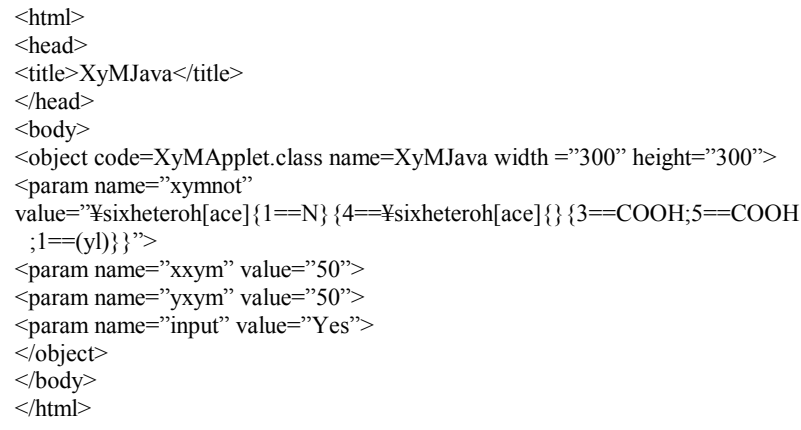

Figure 9. XyM Notation in an HTML Document.

The corresponding formula on a CRT display is shown in Fig. 10, which has been obtained by using the Internet Explorer Macintosh Edition (with MRJ version 1.2.2).

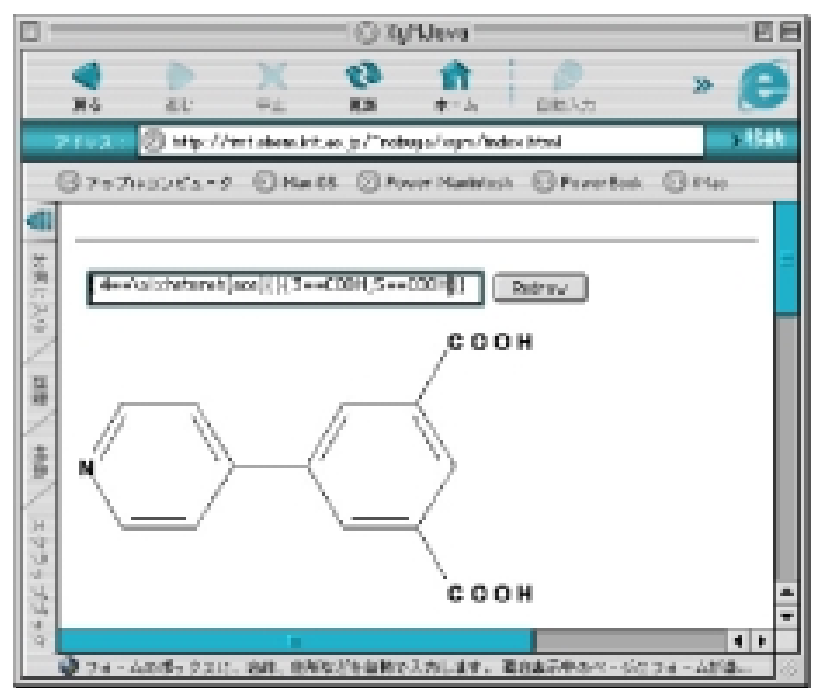

Figure 10. Structural formula on display.

The tags in the HTML document have the following meanings:

1. The Java main class XyMApplet.class is assigned to the attribute 'code' in an <object> tag, which also contains the attribute 'name' to specifiy the XyMJava class and the attributes 'width' and 'height' to set a drawing region for a formula to be drawn.

2. A $<$ param $>$ tag is to set other parameters. The attribute pair 'name=xxym' and 'value' and the pair 'name=yxym' and 'value' respectively specify the $x^{-}$and $y$-coordinate of the bottom-left origin of the drawing region.
3. The attribute pair 'name=xymnot' and 'value' for in a $<$ param $>$ tag designates a XyM notation to be displayed.

4. The attribute pair 'name=input' and 'value $=$ Yes' is used to open a window for key-inputting another XyM notation from a display.

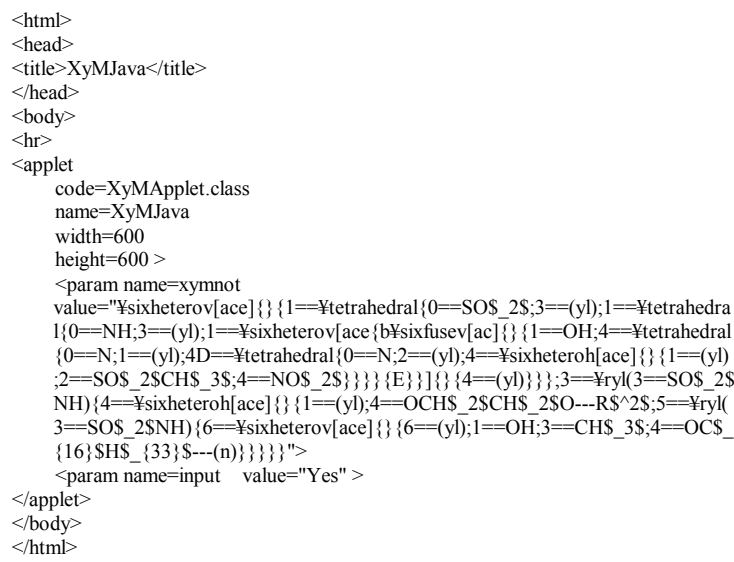

Figure 11. XyM Notation for a Cyan Dye Releaser in an HTML Document.

An HTML document for drawing a complicated example (a cyan dye releaser for instant color photography) is shown in Fig. 11.

The corresponding structural formula is created by using the Windows Internet Explorer 5.5, as shown in Fig. 12. Figures 10 and 12 exemplify a feature that the XyMJava system runs satisfactorily on such representative platforms as Macintosh and Windows machines by virtue of appropriate browser software.

\section{Conclusion}

The XyMJava system has been developed to draw a chemical structural formula by inputting a XyM notation. The object-oriented technique, especially the design-pattern approach, is applied to parse a XyM notation. An HTML document containing a XyM notation can be browsed by the XyMApplet of the XyM Java system, which produces a chemical model for encapsulating information on chemical structural formulas. The chemical model is then used for drawing formulas on a CRT display. Since the parsing class has been implemented as an interface in an abstract fashion, the parsing of any notation or language other than a XyM notation can be implemented without any change of the parsing class. 


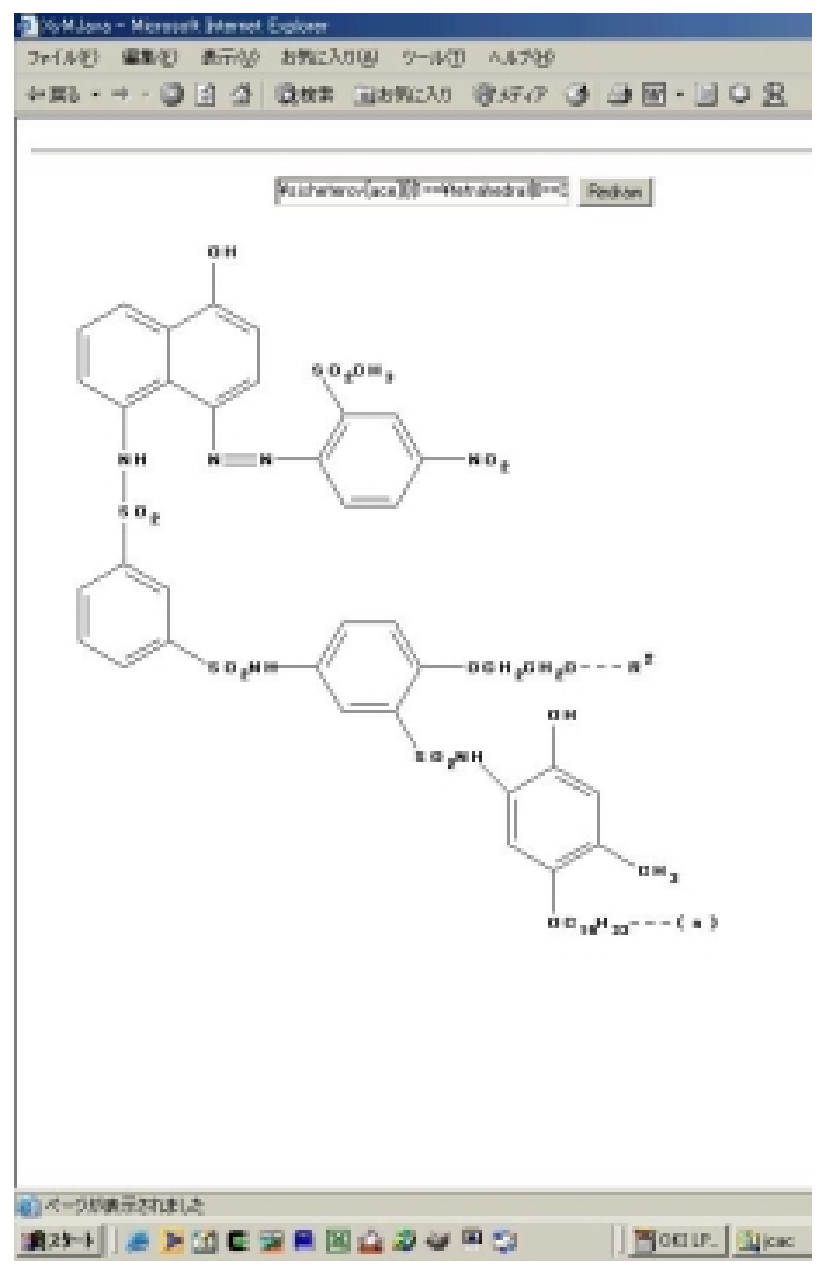

Figure 12. Structural Formula of a Cyan Dye Releaser on Display.

\section{References and Notes}

[1] IUPAC Organic Chemistry Division, Commission on Nomenclature of Organic Chemistry "Nomenclature of Organic Chemistry. Sections A, B, C, D, E, F, and H," 4th ed., Pergamon: Oxford, 1979.

[2] IUPAC Organic Chemistry Division, Commission on Nomenclature of Organic Chemistry (III. 1) "A Guide to IUPAC Nomenclature of Organic Compounds. Recommendations 1993," Blackwell, London, 1993.

[3] Chemical Abstracts "Index Guide 1992-1996, Appendix IV, Chemical Substance Index Names," Chemical Abstracts Service, Columbus, 1997.

[4] K. Hirayama, Kagaku no Ryoiki, 34, 776-783 (1980).

[5] K. Hirayama, "The HIRN System. Nomenclature of Organic Chemistry for Man-to-Machine and
Man-to-Man Communication. Principles," Maruzen, Tokyo and Springer Verlag, Berlin-Heidelberg, 1984.

[6] K. Hirayama, in "Joho kagaku (Information Chemistry), Kagaku Sosetu Vol. 18," ed. by Chemical Society of Japan, Gakkai Shuppan Center, Tokyo 1979, p 9-28.

[7] Y. Kudo, "Joho kagaku (Information Chemistry), Kagaku Sosetu Vol. 18,” ed. by Chemical Society of Japan, Gakkai Shuppan Center, Tokyo, 1979, p $63-90$.

[8] S. Hanai, in "Computer Chemistry," ed. by S. Ono, Maruzen, Tokyo, 1988, p 57-98.

[9] W. J. Wiswesser, J. Chem. Inf. Comput. Sci. 25, $258-263$ (1985).

[10] C. H. Davis and J. E. Rush, "Information Retrieval and Documentation in Chemistry," Greenwood, Westport 1974.

[11] J. E. Ash, P. A. Chubb, S. E. Ward, S. M. Welford, and P. Willett, "Communication, Storage and Retrieval of Chemical Information," Ellis Horwood: Chichester 1985.

[12] National Academy of Sciences-National Research Council "Survey of Chemical Notation Systems," National Academy of Sciences, Washington, D. C. 1964.

[13] National Academy of Sciences "Chemical Structure Information Handling. A Review of the Literature 1962-1968," National Academy of Sciences: Washington, D. C. 1969.

[14] "The Commission on Codification, Ciphering and Punched Card Techniques of the IUPAC Rules for IUPAC Notation for Organic Compounds," John Wiley \& Sons, New York 1961.

[15] G. M. Dyson, in "Chemical Information Systems," ed. by J. E. Ash and E. Hyde, Ellis Horwood, Chichester 1974, p 130-155.

[16] E. G. Smith and P. A. Baker, eds., "The Wiswesser Line-Formula Chemical Notation (WLN)," 3rd ed., Chemical Information Management, Cherry Hill 1976.

[17] H. W. Hayward, H. M. S. Sneed, J. H. Turnipseed, and S. J. Tauber, J. Chem. Doc., 5, 183-189 (1965).

[18] H. Skolnik, J. Heterocyclic Chem., 6, 689-695 (1969).

[19] D. Weininger, J. Chem. Inf. Comput. Sci. 28, $31-36$ (1988)

[20] D. Weininger, A. Weininger, and J. L. Weininger, J. Chem. Inf. Comput. Sci. 29, 97-101 (1989).

[21] D. Weininger, J. Chem. Inf. Comput. Sci. 30, 237-243 (1990).

[22] C. S. Wilcox and R. A. Levinson, in "Artificial Intelligence Applications in Chemistry (ACS Symposium Series 306)," ed. by T. H. Pierree and B. A. Hohne, American Chemical Society, Washington, DC 1986, p 228. 
[23] N. Ghoshal, J. Chem. Inf. Comput. Sci. 30, 308-311 (1990).

[24] S. Ash, M. A. Cline, R. W. Homer, T. Hurst, and G. B. Smith, J. Chem. Inf. Comput. Sci. 37, 71-79 (1997).

[25] C. N. Mooers, Zator Technical Bulletin (Boston: Zator Co., 79 Milk St.)., 59, (1951).

[26] L. C. Ray and R. A. Kirsch, Science 126, 814-819 (1957).

[27] D. J. Gluck, J. Chem. Doc. 5, 43-51 (1962).

[28] L. Spialter, J. Am. Chem. Soc. 85, 2012-2013 (1963).

[29] A. Dalby, J. G. Nourse, W. D. Hounshell, A. K. I. Gushurt, D. L. Greier, B. A. Leland, and J. Laufer, J. Chem. Inf. Comput. Sci. 32, 244-255 (1992).

[30] S. V. Kasparek, "Computer Graphics and Chemical Structures," John Wiley \& Sons, New York 1990.

[31] A. C. Norris and A. L. Oakley, in "TeX-Applications, Uses, Methods," ed. by M. Clark, Ellis Horwood, Chichester 1990, p 207-225.

[32] S. M. Bachrach, ed., "The Internet: A Guide for Chemists," American Chemical Society, Washington, D. C., 1996.

[33] S. Fujita, J. Chem. Inf. Comput. Sci. 39, 915-927 (1999).

[34] The word ' $\mathrm{XyM}$ ' ( $\mathrm{T}_{\mathrm{E}} \mathrm{X}$ as the formal logo) is an uppercase form of the stem $\chi v \mu$ of the Greek word

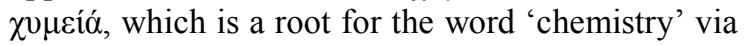
an Arabian word 'alchemy'. The pronuncialtion of XyM is recommended to be 'khým', in which the 'kh' sound may be a Russian ' $k$ ' or more simply an English ' $k$ ' and the symbol ' $y$ ' is expected to be pronounced like a German 'ü'.
[35] P. Muray-Rust and H. S. Rzepa, J. Chem. Inf. Comput. Sci., 39, 928-942 (1999).

[36] S. Fujita, (1993), On-line Manual for XyMTeX Version 1.00. Available from CTAN (tex-archive/macros/latex209/contrib/xymtex/).

[37] S. Fujita, Comput. Chem. 18, 109-116 (1994).

[38] S. Fujita, TUGboats, 16, 80-88 (1995).

[39] S. Fujita, (1996), On-line Manual for XyMTeX Version 1.01.

[40] S. Fujita, "XyMTeX. Typesetting Chemical Structural Formulas," Addison-Wesley Japan, Tokyo 1997.

[41] T. Ogura, M. Nishimoto, and Y. Hatano, Representation of Mathematical Formula, Chemical Formula and Structral Formula on the WWW Browser, in "The 20th Symposium on Chemical Information and Computer Science, Kumamoto," (1997) p 21IP15.

[42] S. Fujita and N. Tanaka, J. Chem. Inf. Comput. Sci. 39, 903-914 (1999).

[43] S. Fujita and N. Tanaka, TUGboat 2000, 21 (1), $7-14$.

[44] G. E. Krasner and S. T. Pope, J. Object-Oriented Programming, 1(3), 26-49 (1988).

[45] B. Frank, "Pattern-Oriented Software Architecture: A System of Patterns," John Wiley \& Sons, New York 1996.

[46] E. Gamma, R. Helm. R. Johnson, J. Vlissides, "Design Pattern Elements of Reusable Object-Oriented Software," Addison Wesley Longman, New York, 1995.

[47] M. Grand, "Patterns in Java, A Catalog of Reusable Design Patterns, Illustrated with UML, Volume 1," John Wiley \& Sons, New York 1998. 


\title{
化学構造式をワールドワイドウェブ伝達するための XyMJavaシステム
}

\author{
田中伸也，藤田眞作 ${ }^{*}$
}

京都工芸纎維大学工芸学部物質工学科, $\overline{0} 606-8585$ 京都府京都市左京区松ヶ崎御所海道町

\begin{abstract}
インターネット上で化学構造式を伝達するための構造式描画システムとしてXyMJavaを開発 した .これは,線形表現のXyM記法を入力言語としており, Javaアプレットとして実装されたシ ステムである.このシステムでは，XyM記法の解析段階で, オブジェクト指向技術，とくにデ ザインパターンの技術を応用している.さらに，構造式情報をカプセル化して画像表示するた めに, 新たに考案した「化学モデル」を採用している.この結果, XyM記法をHTML文書の中 に記入することによって , 化学構造式をインターネットブラウザーで表示することが可能にな った .
\end{abstract}

キーワード：XyM記法, Java, 構造式, WWW

\footnotetext{
*fajitas@chem.kit.ac.jp
} 